\title{
Características neuromotoras e biopsicossocias de lactentes com histórico de risco biológico
}

\section{Motor and biopsychosocial characteristics of nurslings with biological risk bistory}

\author{
Joyce Ribeiro $^{[a]}$, Thais Silva Beltrame ${ }^{[b]}$
}

[a] Fisioterapeuta Especialista em Desenvolvimento Infantil, Bolsista CAPES, Mestre em Ciências do Movimento Humano pela Universidade do Estado de Santa Catarina (UDESC), Florianópolis, SC - Brasil, e-mail: joycefisio@gmail.com

[b] Professora do Departamento de Ciências da Saúde, Coordenadora do Programa de Pós-Graduação em Ciências do Movimento Humano (CEFID), Florianópolis, SC - Brasil, e-mail: bthais@terra.com.br

\section{Resumo}

Introdução: Os avanços tecnológicos têm permitido maior sobrevivência de recém-nascidos de risco. No entanto, os fatores de risco biológico aos quais essas crianças foram expostas fazem com que tenham maior chance de apresentar atrasos no desenvolvimento. Objetivo: O presente estudo objetivou avaliar as características neuromotoras e biopsicossociais de lactentes com histórico de risco biológico. Metodologia: A amostra foi constituída de 25 lactentes com idade desde o nascimento a dois meses de vida, com registro de nascimento na cidade de Fraiburgo, SC. Os fatores de risco biológicos presentes foram: prematuridade, baixo peso, incompatibilidade sanguínea, necessidade de reanimação, intubação, permanência em $\mathrm{O}_{2}$, hipertensão pulmonar leve, icterícia, desconforto respiratório, infecção neonatal, anemia neonatal, tabagismo materno, trauma durante o parto, cardiopatia e má-formação de traqueia. Foram utilizados os seguintes instrumentos: cartão de saúde da criança, formulário biopsicossocial e Neonatal Behavioral Assesment Scale. Resultados: Foi possível constatar, em relação às condições biopsicossociais, prevalência de mães casadas, primigestas, com ensino superior completo, exercendo atividade extra-lar e com idade média de 28 anos na ocasião do parto. Verificou-se casos de exposição do feto ao tabagismo materno, mães acometidas por anemia, hipertensão e hipotensão durante o período gestacional. Em relação ao desenvolvimento neuromotor, os lactentes apresentaram desempenho esperado para crianças de risco em relação à habituação, regulação e reflexos. Em relação à socialização, organização e sistema autônomo, constatou-se desempenho inferior ao de demais crianças de risco. Conclusão: Os achados deste estudo demonstram que crianças com histórico de risco biológico podem apresentar comprometimento em relação a aspectos do desenvolvimento neuromotor.

Palavras-chave: Prematuridade. Baixo peso. Desenvolvimento neuromotor. 


\section{Abstract}

Introduction: The technological advances have allowed a greater survival these newborns at risk. However, the biological risk factors to which these children were exposed make it have a greater chance to present delays in their growth and development. Objective: The present study aimed to evaluate the neuromotor and biopsychosocial characteristics of nurslings with biological risk history. Methods: The sample of the following study was constituted to 25 nurslings with aged from birth to two months of life with register of birth in Fraiburgo, Santa Catarina State. The biological risk factors stated were: prematurity, low weight, sanguine incompatibility, need for resuscitation, intubation, $\mathrm{O}_{2}$ permanence, light lung hypertension, icterus, respiratory discomfort, neonatal infection, neonatal anemia, maternal smoking, trauma during the parturition, cardiopathies and irregular formation of the trachea. For the data collection were used the following instruments: child health card, biopsychosocial form and the Neonatal Behavioral Assesment Scale. Results: Was possible find in the biopsychosocial conditions prevalence of married mothers, first pregnancy, complete higher education, practicing extrahome activity and maternal mean age during the parturition of 28 years old. There were events of fetus exposition to maternal smoking, mothers with anemia, hypertension and hypotension during the gestational period. About the neuromotor development, the nurslings present expected performance for children at risk for habituation, regulation and reflexes. On the socialization, organization and autonomous system it was inferior performance than other children at risk.

Conclusions: The findings of this study argue that children biological risk history can present commitment with respect to aspects of neuromotor development.

Keywords: Prematurity. Low weight. Neuromotor development.

\section{Introdução}

Em várias localidades do Brasil, fatores como baixo peso (BP) ao nascer, prematuridade (PT) e presença de defeitos congênitos estão entre as principais causas de mortalidade neonatal (1). As crianças com BP e prematuras ao nascer apresentam risco de mortalidade significativamente superior a crianças com peso maior ou igual a $2.500 \mathrm{~g}$ e duração da gestação maior ou igual a 37 semanas (2). Além desses fatores de risco biológicos, eventos perinatais e pós-natais adversos, tais como exposição intrauterina do feto a toxicomas, traumas, internação em UTI (Unidade de Terapia Intensiva), submissão a oxigenioterapia e infecções também são fatores que podem interferir na mortalidade e morbidade neonatal (3). Entretanto, nas últimas décadas, muitos avanços tecnológicos ocorreram nas ciências da saúde, permitindo a implantação e implementação de maior número de UTI e melhora dos cuidados perinatais $(4,5,6)$.

Os avanços tecnológicos agregados à capacitação dos profissionais envolvidos em gestações de risco têm permitido maior sobrevivência de recém-nascidos (RN) de risco, proporcionando aumento dessa população $(7,8)$. Esses avanços contribuíram, também, para a melhoria da qualidade de vida dessas crianças e maior acesso aos serviços assistenciais ao parto e ao primeiro ano de vida, tornando mais evidente o prognóstico do desenvolvimento neuropsicomotor à medida que os métodos diagnósticos ficaram mais sofisticados $(2,7,9,10)$. No entanto, apesar do desenvolvimento dos cuidados intensivos permitir maior sobrevivência de RN de risco biológico, a incidência de doenças neurológicas que inviabilizem a sobrevivência dessas crianças em condições adequadas não tem se modificado significativamente $(5,4,11)$.

Os fatores de risco biológico aos quais essas crianças foram expostas as fazem ter maior chance de apresentar atrasos no seu potencial de crescimento e desenvolvimento $(11,12)$. Apesar de sequelas graves estarem diminuindo consistentemente em bebês de risco, há evidências de atrasos e 
distúrbios leves do desenvolvimento entre sobreviventes, fato que torna necessário o acompanhamento cuidadoso desses bebês, uma vez que apresentam maior vulnerabilidade em relação às alterações no desenvolvimento neuropsicomotor $(9,13,14)$.

Com base no anteriormente exposto, formulou-se a seguinte questão: Quais as características biopsicossociais e neuromotoras de lactentes com histórico de risco biológico?

\section{Método}

Esta pesquisa caracteriza-se como uma pesquisa descritiva diagnóstica do tipo transversal. Cumprindo os termos da Resolução 196/96 e 251/97 do Conselho Nacional de Saúde (Número de Referência 193/2007), esta pesquisa obteve parecer favorável do Comitê de Ética em Pesquisa em Seres Humanos da Universidade do Estado de Santa Catarina (UDESC).

A população deste estudo foi representada por todas as crianças com idade compreendida desde o nascimento aos dois meses de vida, que durante o período de coleta de dados (de abril a setembro de 2008) apresentou registro de nascimento nas unidades de Saúde do município de Fraiburgo, SC. No ano de 2008 (até o mês de agosto) houve registro de 350 nascimentos, dos quais 22 apresentaram BP ao nascimento.

A seleção da amostra foi realizada de forma aleatória, tendo os seguintes critérios de inclusão: apresentar registro de nascimento na cidade de Fraiburgo, SC; possuir o termo de consentimento assinado pelo responsável da criança; ter idade compreendida entre o nascimento e os dois meses de vida e apresentar histórico de fatores de risco biológicos tais como: prematuridade, crescimento intrauterino retardado, defeitos congênitos, asfixia perinatal e encefalopatia hipóxica-isquêmica, hemorragia intracraneal, displasia broncopulmonar, anormalidades bioquímicas (hipoglucemia, policitemia, hiperbilirrubinemia), infecções neonatais, crises convulsivas neonatais, exploração neurológica anormal e abusos de substâncias tóxicas por parte da mãe no período gestacional e traumas encefálicos durante o parto. A identificação das crianças que apresentavam histórico de risco era dada pelo registro de nascimento, pelo cartão de saúde da criança ou, no caso de exposição de toxicomas, informado pela mãe.

A amostra do seguinte estudo foi constituída de 25 lactentes, sendo 16 do sexo masculino e 9 do sexo feminino. Quanto aos fatores de risco biológicos presentes no grupo controle, constatouse: 4 casos $(5,0 \%)$ de P'T e BP e alguma intercorrência associada pós-parto, tais como necessidade de reanimação, intubação, permanência em $\mathrm{O}_{2}$, hipertensão pulmonar leve, icterícia, desconforto respiratório, infecção neonatal, anemia neonatal, 6 casos $(7,5 \%)$ de PT associado a intercorrências, 2 casos $(2,5 \%)$ de PT, dois casos (2,5\%) de BP, 4 casos de tabagismo materno (5,0\%), 4 casos de trauma durante o parto (bossa) (5,0\%) e 3 casos (3,8\%) de demais intercorrências (1 caso de cardiopatia, 1 caso de má-formação de traqueia e 1 caso de icterícia associado a incompatibilidade).

Apresentavam idade compreendida do nascimento aos 15 dias durante o período da avaliação 8 lactentes, 5 tinham idade entre 16 e 31 dias, 9 entre 32 e 47 dias e 3 entre 48 e 63 dias. As crianças que apresentaram prematuridade tiveram Idade Cronológica (IC) corrigida para a termo (14).

\section{Instrumentos de coleta de dados}

\section{Cartão de saúde da criança}

Informações sobre antecedentes clínicos foram coletadas a partir do Cartão de Saúde da Criança proposto pelo Ministério da Saúde. O Cartão da Criança contém informações referentes ao estado da criança desde o nascimento, peso ao nascimento, comprimento, PC, Apgar, dentre outras informações relevantes, assim como traz informações de intercorrências, quando presentes. Essas informações foram registradas no Formulário Biopsicossocial. 


\section{Formulário Biopsicossocial}

O Formulário Biopsicossocial foi criado com o objetivo de registrar informações referentes aos dados pessoais de cada criança avaliada, dados gestacionais, dados do nascimento e dados pósnatais, além de informações sobre o estado de saúde da criança no dia da realização da avaliação, horário da última mamada, horário que acordou, peso e estatura, estado de alerta, entre outros fatores relevantes.

Os dados referentes à identificação do bebê e os dados gestacionais, como doenças ou anormalidades apresentadas pela mãe durante a gestação, ingestão de medicamentos e substâncias tóxicas e exposição ao RX, foram informados pela mãe do lactente, bem como informações referentes à amamentação e informações sobre o estado de saúde da criança no dia da avaliação motora. Essas informações foram coletadas por entrevista com a mãe antes do início da avaliação motora do lactente e registradas no Formulário Biopsicossocial.

\section{Neonatal Behavioral Assesment Scale}

A Neonatal Behavioral Assesment Scale (Escala de Brazelton) é uma técnica para avaliação neonatal que permite avaliação específica e objetiva de diferentes características

neuromotoras. É considerado atualmente o mais sensitivo instrumento para detectar as diferenças neurocomportamentais em RN (15).

A escala consiste de dois tipos de itens: neurocomportamentais e reflexos. A primeira parte é composta de 35 variáveis que retratam aspectos sobre as ações e reações do bebê. Todos os itens são divididos em grupos de acordo com parâmetros: Habituação (mensura habilidade de se habituar a estímulos), Social Interativo ou Orientação (mensura habilidade de atender a estímulos visuais, auditivos e à qualidade de alerta apresentada durante os estímulos), Sistema Motor (mensura performance motora e qualidade do movimento e do tônus), Estado de Organização (mensura nível de excitabilidade e labilidade), estado de Regulação (capacidade de a criança regular seu estado de consciência com aumento de estímulos), Sistema Autônomo (registra sinais de estresse relacionados a ajustes homeostáticos do SNC). As respostas de reflexos são avaliadas utilizando-se as respostas a 18 itens, o número de reflexos anormais é registrado (15).

Todos os itens comportamentais são avaliados em uma escala de 1 a 9 pontos (sendo 9 a melhor performance), exceto 8 itens da escala, que, de acordo com o Manual, têm recordes lineares na pontuação 5, 6 ou 8 (15).

\section{Procedimento de coleta e análise dos dados}

Primeiramente, os objetivos desta pesquisa, bem como procedimentos metodológicos, foram explanados à Secretária da Saúde da Cidade de Fraiburgo, SC, verificando a viabilidade da realização do estudo na referida cidade. Após obtenção da aprovação da Secretaria da Saúde de Fraiburgo, o projeto foi submetido à avaliação do Comitê de Ética. Após determinação da unidade onde seria desenvolvido o estudo, o pesquisador passou a frequentar esta unidade e realizar as avaliações na sala do SISVAN, onde era feita a avaliação pondo estatural dos lactentes, sendo assim, as crianças compareciam ao posto para vacinação e posteriormente eram encaminhadas ao SISVAN para acompanhamento pondo estatural, para então serem avaliadas pela fisioterapeuta e depois pesadas e mensuradas pela técnica de enfermagem responsável do setor.

As crianças eram avaliadas pela fisioterapeuta somente após obtenção da assinatura do termo de consentimento livre pelo responsável. Primeiramente, era realizada a verificação do Cartão de Saúde da Criança e entrevista com a mãe, e posteriormente realizada a avaliação neuromotora. 


\section{Tratamento estatístico}

Os dados referentes às condições biopsicossociais e características neuromotoras foram tratados por meio da estatística descritiva (média, desvio-padrão, valores máximos e mínimos) usando o programa SPSS versão 13.0 .

\section{Resultados}

\section{Condições biopsicossociais}

Quanto ao estado civil, $8(32 \%)$ mães eram solteiras, 9 (36\%) casadas e $8(32 \%)$ moravam com o pai do bebê. A maioria das mães era primigesta $(n=14,56 \%)$. Quanto ao tipo de parto, houve prevalência de parto cesáreo $(n=18,72 \%)$, o parto induzido foi realizado em um caso $(4 \%)$. A maioria das mães exerciam algum tipo de atividade extra-lar $(n=14,56 \%)$ e tinham ensino superior completo $(\mathrm{n}=10,40 \%)$.

Em relação à faixa etária materna na ocasião do parto, as maiores proporções foram encontradas na idade entre 19 e 23 anos, com 9 mães (36\%), e entre 34 e 38 anos, com 6 mães (24\%). A idade materna na ocasião do parto variou dos 14 aos 40 anos.

Quanto às doenças da mãe durante a gestação, a anemia apresentou-se em número significativo, acometendo 8 mães (32\%). Apresentaram hipertensão 4 mães $(16 \%)$ e hipotensão 7 mães $(28 \%)$. No que diz respeito à ingestão de substâncias tóxicas na gestação, 6 mães $(7,5 \%)$ relataram ter fumado durante a gestação. O uso de outros tipos de substâncias tóxicas (álcool, drogas) não foi relatado por nenhuma mãe do presente estudo.

O valor médio do índice de Apgar no primeiro minuto foi de 7,00 (dp $\pm 2,3$ ), apresentando valor mínimo de 3 e máximo de 9. Quanto ao Apgar no quinto minuto, a média foi de 8,6 (dp $\pm 0,86$ ), tendo valor mínimo de 6 e máximo de 9. A média de peso no nascimento foi $2,783 \mathrm{~kg}$ (dp $\pm 556,80)$.

\section{Características neuromotoras}

Os dados referentes ao desenvolvimento neuromotor dos lactentes de risco biológico estão explanados na Tabela 1, representados por seus valores médio, máximo, mínimo e desvio-padrão.

Tabela 1 - Características Neuromotoras

\begin{tabular}{lccccc}
\hline & N & Mínimo & Máximo & Média & 土p \\
\hline Habituação & 7 & 3,00 & 7,25 & 5,35 & 1,36 \\
Social/Orientação & 24 & 2,00 & 7,28 & 4,98 & 4,38 \\
Motricidade & 25 & 2,42 & 6,20 & 2,22 & 0,88 \\
Organização & 25 & 1,00 & 3,75 & 5,54 & 0,90 \\
Regulação & 25 & 2,24 & 8,25 & 3,94 & 0,72 \\
S. Autônomo & 25 & 1,00 & 10,00 & 4,28 & 1,36 \\
Reflexos & 25 & 0,00 & & & \\
\hline
\end{tabular}


Pode-se observar que um número relevante de lactentes não realizou os testes de habituação. Este fato também ocorreu em outros estudos que utilizaram o mesmo instrumento de avaliação. No estudo de Fenster (16), por exemplo, mais de metade das crianças participantes de seu estudo (53\%) não realizaram todos os itens do pacote de testes de habituação por causa da exigência de que a criança deve estar dormindo imediatamente antes da avaliação e ainda permanecer neste estado durante a avaliação.

Os reflexos primitivos com pontuação considerada anormal apresentaram-se nas seguintes proporções: preensão palmar em 20\%, babinsk em 4\% dos casos, clônus em 4\% dos casos, rotação em $48 \%$ dos casos, sucção em $28 \%$ dos casos, glabela em 12\% dos casos, resistência passiva das penas em $24 \%$ dos casos, resistência passiva dos braços em 28\% dos casos, preensão plantar em 16\% dos casos, extensão de MMII em 22\% dos casos, flexão e extensão de MMII em 28\% dos casos, marcha em $22 \%$ dos casos, arrastar em 60\%, gallant em 56\% dos casos, TNR (24\% casos) e Moro em $48 \%$.

\section{Discussão}

\section{Condições biopsicossociais}

A proporção de mães adolescentes (idade inferior a 20 anos) e mães com idade superior a 30 anos, segundo Azenha et al. (17), aumentou significativamente nos últimos anos, fato este constatado no presente estudo visto que um número significativo de mães apresentou-se nas condições citadas por Azenha. A idade materna inferior aos 20 anos, segundo Azenha et al. (17) e Chalem et al. (18), traz risco tanto para a mãe quanto para o bebê, aumentando a chance de BP ao nascimento. Estudiosos fazem menção à associação de que idade materna igual ou superior a 35 anos, presente em proporções significativas neste estudo, está associada a resultados perinatais adversos $(19,20)$.

Neste estudo foram encontrados casos de tabagismo materno, porém não houve relato de exposição do bebê a algum outro tipo de substância tóxica no período gestacional. Dados diferenciados foram encontrados nos estudos de Young et al. (21), nos quais foram encontradas proporções de 5\% de mães fumantes, porém os autores contataram proporções elevadas de uso de álcool (14\% e 16\%, respectivamente) durante a gestação.

Em relação aos índices de Apgar, dos 40 bebês prematuros participantes do estudo de Olhweiler, Silva e Rotta (11), 32,5\% tiveram Apgar entre 8 e 9 no quinto minuto, 5\% apresentaram Apgar 7 e 10\% ficaram com Apgar 10, dado este semelhante ao encontrado no referido estudo onde as médias foram maiores que 7 tanto no primeiro quanto no quinto minuto.

Quanto ao peso no nascimento, nasceram com menos de 2500 gramas 8 (32\%) lactentes, segundo Kassar et al. (22), crianças que nascem com peso inferior a $2500 \mathrm{~g}$ (baixo peso ao nascer - BPN) e com peso situado entre $2500 \mathrm{~g}$ e $2999 \mathrm{~g}$ (peso insuficiente) estão sob alto risco de morrer ou adoecer.

Em relação ao comprimento dos lactentes ao nascimento, Pedro e Rotta (23) constataram comprimento que variou de $44 \mathrm{~cm}$ a $54 \mathrm{~cm}(\mathrm{dp} \pm 1,8)$ em suas pesquisas, tendo média de 49,49 $\mathrm{cm}$, valor um pouco diferenciado ao encontrado neste estudo, visto que a média de comprimento ao nascimento foi de $46,04 \mathrm{~cm}(\mathrm{dp} \pm 2,56)$.

Em relação às características neuromotoras, observa-se na Tabela 1 que o valor médio apresentado pelo grupo de risco quando aos testes de habituação foi de 5,35, esta pontuação corrobora com a encontrada por demais pesquisadores que estudaram o desenvolvimento neuromotor de lactentes de risco $(24,25)$. Os participantes do estudo de Reif et al.(25) apresentaram baixas pontuações em relação ao pacote de habituação por causa da influência das alterações químicas decorrentes da depressão materna. Segundo Reif et al. (25), lactentes de mãe com histórico de depressão são mais lentos em relação à habituação e menos atentos que lactentes de mães não acometidas pela depressão.

A habituação é um importante componente do comportamento dos neonatos que lhes permite habituarem-se à eventos perturbadores, ou seja, permite que eles sejam capazes de aprender a habituar-se a estímulos que seriam capazes de desorganizar o seu comportamento (15). A presença da habituação 
representa que o lactente é capaz de se autoproteger do ambiente e viver confortavelmente por habituar-se a estímulos táteis, auditivos e visuais. Segundo menção de Allin et al. (26), fatores tais como BP e PT podem afetar as funções neurológicas e cognitivas do lactente, acarretando atraso no seu neurodesenvolvimento.

Em relação à orientação/socialização, o valor médio apresentado pelo grupo de risco biológico foi de 4,9. Segundo Brazelton e Nugent (15), a orientação representa a capacidade do lactente de atender a estímulos tanto visuais como auditivos, bem como à qualidade de vigilância que o lactente apresenta durante a apresentação das respostas a esses estímulos. Em relação à pontuação apresentada pelo grupo de risco quanto a orientação/socialização, a mesma pontuação média foi encontrada por Reif et al. (25) ao avaliarem a influência da depressão materna no desenvolvimento neuromotor do lactente. Porém, outros estudos que verificaram o desenvolvimento da socialização/orientação de lactentes expostos a situações de risco constataram um desempenho melhor do que o apresentado pela amostra de risco do presente estudo e pelo estudo de Field et al. (27). Ao avaliar mães com depressão, Field, Diego e Reif (24) constataram valores médios de 6,0 na socialização dos lactentes avaliados. Young (21) avaliou o desenvolvimento neuromotor de lactentes expostos à dichlorodiphenyltrichloroethane (DDT) e constatou valor médio de 7,5 em relação à orientação.

Em relação à motricidade, o valor médio apresentado pelo grupo de risco foi de 4,3, valor que se assemelha aos dados encontrados nos estudos de demais pesquisadores $(24,28)$.

Cooper e Ratcliffe (28) avaliaram a motricidade de lactentes com histórico de PT e constataram valor médio de 4,26 em relação à motricidade. Morrow et al. (29) também constataram desempenho semelhante em relação à motricidade de lactentes expostos ao tabagismo durante o período gestacional, apresentado valor médio de 4,5. Field, Diego e Reif (24) constataram valor médio de 4,0 em relação à motricidade de lactentes filhos de mães depressivas.

A população investigada pelos referidos pesquisadores se assemelham significativamente com a população deste estudo em relação ao desempenho motor e em relação ao tipo de risco biológico apresentado, quando comparada com os estudos de demais pesquisadores $(28,29)$.

Ao avaliar o desenvolvimento neuromotor de lactentes, Gregório et al. (6) avaliaram 281 lactentes com histórico de risco biológico, mais especificamente lactentes com histórico de BP ao nascimento e constataram que $40 \%$ dessas crianças apresentaram alterações neuromotoras transitórias nos primeiros meses de vida. Segundo Brazelton e Nugent (15), o nível de atividade motora ou desempenho motor do lactente representa a maturidade motora do lactente.

Referente ao desempenho dos lactentes com histórico de risco biológico no que diz respeito à organização, a pontuação média foi de 2,2, pontuação esta inferior à encontrada por demais pesquisadores ao verificar o desempenho de lactentes de risco quanto à organização. Morrow et al. (29) avaliaram lactentes expostos ao tabagismo materno durante a gestação e constataram pontuação média de 3,7. Cooper e Ratcliffe (28) avaliaram lactentes prematuros e constataram pontuação de 4,0. Portanto, os dados encontrados pelos pesquisadores representam um desempenho inferior dos lactentes de risco avaliados pelo presente estudo. Segundo Brazelton e Nugent (15), a organização representa comportamentos do lactente, tais como irritabilidade, piscos de excitação e mudanças de comportamento durante certo período de tempo. Essas atividades, segundo os autores, são indispensáveis e úteis para a aprendizagem do lactente sobre o meio ambiente e sobre si mesmos e interferem significativamente no desenvolvimento do lactente.

A regulação a pontuação média apresentada pelos lactentes de risco foi de 5,5. Este dado foi semelhante ao encontrado por demais pesquisadores ao verificar o desempenho de lactentes de risco em relação à regulação, tais como os estudos de demais pesquisadores (16, 21, 24), nos quais constataram-se os seguintes valores médios, respectivamente, 5,7; 5,7 e 5,6. É por meio da regulação que o lactente mantém o equilíbrio em relação a seus componentes químicos e processos fisiológicos (30). Brazelton e Nugent (15) relatam que a regulação representa aspectos do comportamento do lactente representados pelo choro, pela quantidade de estímulos necessários para que ele chore, pela quantidade de esforço necessária para que ele pare de chorar ou ele mesmo se console.

Em relação ao sistema autônomo dos lactentes, este representa a integridade fisiológica do recém-nascido, os lactentes que apresentam menor pontuação são mais frágeis e demonstram 
maior instabilidade autonômica, pelas mudanças de coloração da pele, tremores, alterações respiratórias, entre outros comportamentos relacionados ao sistema autônomo é que a integridade do sistema autônomo do lactente pode ser percebida segundo Brazelton e Nugent (15). A média apresentada pelos lactentes de risco em relação ao sistema autônomo foi de 3,9, desempenho este inferior ao encontrado por demais pesquisadores.

Avaliou o desempenho do sistema autônomo de lactentes filhos de mães depressivas (24, 27), os pesquisadores constaram pontuações que representam um desempenho superior do que o constatado no presente estudo (6,0 e 5,8 respectivamente). O desempenho médio de lactentes com histórico de PT avaliados por Cooper e Ratcliffe (28) foi de 5,2.

Quanto ao comportamento motor reflexo, pode-se observar na Tabela 1 que os lactentes de risco biológico do presente estudo apresentaram valor médio bastante significativo em relação aos reflexos. Quanto maior a pontuação em relação aos reflexos pior é o desempenho da criança, visto que todos os reflexos que a escala de Brazelton avalia devem estar presentes até os dois meses, com intensidade adequada, bem como simetria. A pontuação ideal seria 0 , porém pode-se observar que no grupo de risco do presente estudo, constatou-se valor mínimo de 3,00, valor médio de 5,3 e máximo de 10,00, conforme apresentado na Tabela 1.

Quando comparado o resultado deste estudo com o de demais pesquisadores, pode-se verificar que Morrow et al.(29) constataram como pontuação média em relação aos reflexos de lactentes expostos ao tabagismo materno o valor de 5.2.

Lactentes avaliados, após três dias de vida, por Young et al. (21) apresentaram mais de três reflexos anormais em 17\% dos casos. Segundo Futagy, Otani e Imai (31), uma resposta anormal de um reflexo pode refletir em uma disfunção do SNC, não apenas na coluna vertebral, mas também nos níveis mais elevados do córtex. No estudo de Fenster et al. (16) foi encontrado uma prevalência de 20,8\% dos lactentes que apresentavam quatro ou mais reflexos anormais.

\section{Conclusão}

Constatou-se em relação às condições biopsicossociais prevalência de mães casadas, com idade média de 28 anos na ocasião do parto e que exerciam atividade remunerada. Identificou-se casos de anemia, alterações pressóricas, diabetes, sífilis e hemorragias entre as patologias que acometeram as mães durante o período gestacional. Em relação às características neuromotoras, os lactentes apresentaram desenvolvimento neuromotor diferenciado de demais crianças de risco em relação à socialização, organização e sistema autônomo, demonstrando déficits.

Este fato torna necessária a menção de que esses lactentes necessitam ser acompanhados por programas estruturados de avaliação e intervenção neuromotora, visto que possíveis déficits no desenvolvimento nesta idade, podem interferir no desenvolvimento psicomotor em idade subsequente, trazendo consequências em relação à interação da criança com o ambiente, promovendo alterações secundárias que poderão repercutir em seu desempenho em atividades de vida diária, comprometendo a independência e qualidade de vida desses lactentes.

Também sugere-se que as condições de estimulação motora apresentadas a essas crianças sejam verificadas e implementadas pelo fisioterapeuta, visto que condições ambientais agregadas às condições de risco biológicos podem ser determinantes no desenvolvimento neuromotor.

\section{Referências}

1. Oliveira CA, Lins CP, Sá RAM, Netto HC, Bornia RG, Silva NR, et al. Síndromes hipertensivas da gestação e repercussões perinatais. Rev Bras Saúde Mater Infant. 2006;6(1):93-98.

2. Kilsztajn S, Rossbach A, Carmo MSN, Sugahara GTL. Assistência pré-natal, baixo peso e prematuridade no Estado de São Paulo, 2000. Rev Saúde Pública. 2003;37(3):303-10. 
3. Vargas LED, Saénz RCF, Pérez LJ, López SA, Santano GMR, González-Meneses A. Deficiencias: medidas preventivas durante el período neonatal. Vox Paediatrica 1999;7(1):81-90.

4. Resegue R, Puccini R, Silva E. Risk factors associated with developmental abnormalities among high-risk children attended at a multidisciplinary clinic. Sao Paulo Med J. 2008;126(1):4-10.

5. Hack M, Taylor HG. Perinatal brain injury in preterm infants and later neurobehavioral function. JAMA. 2000;284(15):1973-4.

6. Gregório CSB, Pinheiro ECT, Campos DEO, Alfaro EJ. Evolução neuromotora de um recém-nascido prétermo e a correlação com os fatores perinatais. Fisioterapia Brasil. 2002;3(4):250-7.

7. Barbosa VC, Formiga CKMR, Linhares MBM. Avaliação das variáveis clínicas e neurocomportamentais de recém-nascidos pré-termo. Rev Bras Fisioter. 2007;11(4):275-81.

8. Santos RS, Araújo APQC, Porto MA. Diagnóstico precoce de anormalidades no desenvolvimento em prematuros: instrumentos de avaliação. J Pediatr (Rio J.). 2008;84(4):289-99.

9. Magalhães LC, Barbosa VM, Lopes KC, Paixão ML. Estudo longitudinal do desenvolvimento do recémnascido pré-termo avaliação na idade pré-escolar. Rev Bras Neurol. 1999;35(4):87-93.

10. Almeida MFB, Guinsburg R, Martinez FE, Procianoy RS, Leone CR, Marba STM, et al. Fatores perinatais associados ao óbito precoce em prematuros nascidos nos centros da Rede Brasileira de Pesquisas Neonatais. J Pediatr (Rio J.). 2008;84(4):300-7.

11. Olhweiler L, Silva AR, Rotta NT. Estudo dos reflexos primitivos em pacientes recém-nascidos pré-termo normais no primeiro ano de vida. Arq Neuro-Psiquiatr. 2005;63(2a):294-7.

12. Sabates A, David E. Infant's growth and development assessment in the presence of congenital cardiopathies - a descriptive study. Online Brazilian J Nursing. 2006;5(2):1-15.

13. Burns Y, O’Callaghanb M, McDonella B, Rogers Y. Movement and motor development in ELBW infants at 1 year is related to cognitive and motor abilities at 4 years. Early Hum Dev. 2004;80(1):19-29.

14. Rugolo LM. Crescimento e desenvolvimento a longo prazo do prematuro extremo. J Pediatr (Rio J). 2005;81(1 Supl):S101-S110.

15. Brazelton TB, Nuget JK. Neonatal behavioral assessment scale. London: MacKeith Press; 1995.

16. Fenster L, Eskenazi B, Anderson M, Bradman A, Hubbard A, Barr DB. In utero exposure to DDT and performance on the brazelton neonatal behavioral assessment scale. Neurotoxicology. 2007;28(3):471-7.

17. Azenha VM, Mattar MA, Cardoso VC, Barbieri MA, Del CLA, Bettiol H. Peso insuficiente ao nascer: estudo de fatores associados em duas coortes de recém-nascidos em Ribeirão Preto, São Paulo. Rev Paul Pediatr. 2008;26(1):27-35.

18. Chalem E, Mitsuhiro SS, Ferri CP, Barros MCM, Guinsburg R, Laranjeira R. Gravidez na adolescência: perfil sóciodemográfico e comportamental de uma população da periferia de São Paulo, Brasil. Cad Saúde Pública. 2007;23(1):177-86.

19. Guimarães EAA, Melendez GV. Determinantes do baixo peso ao nascer a partir do sistema de informação sobre nascidos vivos em Itaúna, Minas Gerais. Rev Bras Saude Mater Infant. 2002;2(3):283-90.

20. Senesi LG, Tristão EG, Andrade RP, Krajden ML, Oliveira JFC, Nascimento DJ. Morbidade e mortalidade neonatais relacionadas à idade materna igual ou superior a 35 anos, segundo a paridade. RBGO. 2004;26(6):47782. [acesso 16 dez. 2008 ]. Disponível em: http://www.scielo.br/scielo

21. Young JG, Eskenazi B, Gladstone EA, Bradman A, Pedersen L, Johnson C, et al. Association between in utero organophosphate pesticide exposure and abnormal reflexes in neonates. Neurotoxicology. 2005;26(2):199-209. 
22. Kassar SB, Gurgel RQ, Albuquerque MFM, Barbieri MA, Lima MC. Peso ao nascer de recém-nascidos de mães adolescentes comparados com o de puérperas adultas jovens. Rev Bras Saude Mater Infant. 2005;5(3):293-9.

23. Pedroso FS, Rotta NT. Neurological examination in the healthy term newborn. Arq Neuropsiquiatr. 2003;61(2A):165-9.

24. Field T, Diego M, Reif MH. Prenatal dysthymia versus major depression effects on the neonate. Infant Behav Dev. 2008;31(2):190-3.

25. Reif MH, Field T, Diego M, Ruddock M. Greater arousal and less attentiveness to face/voice stimuli by neonates of depressed mothers on the brazelton neonatal behavioral assessment scale. Infant Behav Dev. 2006;29(4):594-8.

26. Allin M, Rooney M, Griffiths T, Cuddy M, Wyatt J, Rifkin L, et al. Neurological abnormalities in young adults born preterm. J Neurol Neurosurg Psychiatry. 2006;77(4):495-9.

27. Field T, Reif MH, Diego M, Figueiredo B, Schanberg S, Kuhn C. Prenatal cortisol, prematurity and low birthweight. Infant Behav Dev. 2006;29(2):268-75.

28. Medoff-Cooper B, Ratcliffe SJ. Development of preterm infants feeding behaviors and brazelton neonatal behavioral assessment scale at 40 and 44 Weeks' Postconceptional Age. ANS Adv Nurs Sci. 2005;28(4):35663.

29. Morrow CR, Bandstra ES, Anthony J.C, Ofir AY, Xue L, Reyes ML. Influence of prenatal cocaine exposure on full-term infant neurobehavioral functioning. Neurotoxicol Teratol. 2001;23(6):533-44.

30. Diego M, Field T, Reif MH. Prepartum, postpartum and chronic depression effects on neonatal behavior. Infant Behav Dev. 2005;28(2):155-64.

31. Futagy Y, Otani K, Imai K. Asymmetry in plantar grasp response during infancy. Pediatr Neurol. 1995;12(1):54-7.

Recebido: 26/01/2009

Received: 01/26/2009

Aprovado: 10/09/2009

Approved: 09/10/2009 